\title{
Níveis dos neurotransmissores estriatais durante 0 estado epiléptico
}

\author{
Rivelilson Mendes de Freitas ${ }^{1}$ \\ Glauce Socorro Barros Viana² \\ Marta Maria de França Fonteles ${ }^{2}$
}

Recebido: 14/2/2003 Aceito: 12/5/2003

\begin{abstract}
RESUMO
O objetivo desse estudo foi verificar os níveis dos neurotransmissores estriatais de ratas adultas durante o estado epiléptico induzido pela pilocarpina. Ratas wistar foram tratadas com uma única dose de pilocarpina (400 mg/ $\mathrm{kg}$ por via subcutânea (S.C.); P400) e os controles receberam salina. A concentração dos neurotransmissores foi determinada através do HPLC eletroquímico, no corpo estriado de ratas que no período de observação de 1 hora desencadearam estado epiléptico e que sobreviveram à fase aguda do quadro convulsivo. Foi observada redução nos níveis de dopamina, serotonina, ácido diidroxifenilacético e aumento na concentração do ácido 5-hidroxiindolacético. Nenhuma alteração foi observada no 4-hidroxi-3-metoxi-fenilacético. Os resultados sugerem que a ativação do sistema colinérgico pode interagir com os sistemas dopaminérgico e serotonérgico nos mecanismos referentes à fase aguda do processo convulsivo no corpo estriado de ratos desenvolvidos.
\end{abstract}

Unitermos: Dopamina; Serotonina; Convulsão; Corpo estriado.

\section{ABSTRACT}

\section{Striatal monoamines levels during status epilepticus}

The purpose of the present work to investigate the striatal neurotransmissors level in adult rats after status epilepticus induced by pilocarpine. Wistar rats were treated with a single dose of pilocarpine $(400 \mathrm{mg} / \mathrm{kg}$; s.c.; P400) and the controls received saline. Adult animals were closed observed for behavioural changes during $1 \mathrm{~h}$. In this period, the animals that developed status epilepticus and survive this acute phase of seizures had the brains removed and striatal neurotransmissors level determiden by HPLC. The concentration of dopamine, serotonine, dihydroxyphenylacetic acid was reduced and an concentration increase in 5-hydroxyindolacetic acid. Didn't observed alteration in 4-hydroxy3-methoxy-phenylacetic acid. These results suggest that cholinergic activation can interage with dopaminergic and serotonergic systems in acute phase of the convulsive process in rat mature striatum.

Keywords: Dopamine; Serotonine; Seizures; Striatum.

Departamento de Fisiologia e Farmacologia da Universidade Federal do Ceará (UFC), Fortaleza, CE.

Doutorando em Farmacologia.

Professora Adjunta e Pesquisadora do Laboratório de Neurofarmacologia.

Endereço para correspondência:

Rivelilson Mendes de Freitas

Departamento de Farmácia e Laboratório de Neurofarmacologia do Departamento de Fisiologia e Farmacologia da Universidade Federal do Ceará (UFC).

Rua Frederico Severo, 201, ap. 103/B1 07 - Messejana, Fortaleza - CEP 60830-310

Fone: (85) 288-8337 - Fax: (85) 288-8257

E-mail: rivmendes@bol.com.br 


\section{Introdução}

A epilepsia é um distúrbio neurológico com taxa de prevalência de $5 \%$. O estado epiléptico é uma forma severa de contínuos ataques de grande mal da epilepsia em humanos (Aminoff e Simon, 1980) e constitui uma emergência médica associada a alta taxa de mortalidade. As seqüelas mais comuns do estado epiléptico compreendem as convulsões recorrentes espontâneas, os déficit neurológico permanente e a disfunção intelectual. O modelo de epilepsia com dose alta de pilocarpina permite investigar o processo convulsivo mediante alterações comportamentais, eletroencefalográficas e pela presença de lesão cerebral (Clifford et al., 1987; Persinger et al., 1993; Marinho et al., 1997), que são semelhantes à epilepsia do lobo temporal em humanos, permitindo também o estudo das alterações em nível de receptores (Marinho et al., 1998), e/ou dos níveis das monoaminas e seus metabólitos durante ao estado epiléptico (El-Etri et al., 1993; Khan et al., 2000).

A ativação do receptor colinérgico muscarínico (Persinger et al., 1993; Marinho et al., 1998) foi sugerida como responsável pelas convulsões produzidas pela pilocarpina, dando a entender que após ativação do sistema colinérgico haveria uma interação direta e/ou indireta com outros sistemas, a saber: dopaminérgico (Al-Tajir et al., 1990a; Barone et al., 1991), glutamatérgico (Fujikawa et al., 1994 e 1995) e GABAérgico (Fritschy et al., 1999; Erakovic et al., 2000; CostaLotufo et al., 2002), que podem ser ativados para a manutenção e/ou propagação das convulsões. Contudo, os neurotransmissores e as estruturas cerebrais envolvidas na convulsão precisam ser prontamente esclarecidos, a fim de contribuir para a compreensão da gênese do estado epiléptico, bem como investigar novos agentes terapêuticos.

O estudo do cérebro desenvolvido, com suas inervações e densidade de receptores operantes, favorece o conhecimento da epilepsia em relação ao estudo das convulsões em animais adultos (George e Kulkarni, 1996), uma vez que pode ajudar na investigação dos mecanismos de manutenção e propagação das convulsões, ainda ausentes em ratos jovens, podendo ainda indicar as possíveis alterações nas concentrações dos neurotransmissores (Erakovic et al., 2000) que ocorreram no estriado maduro durante o estado epiléptico.

A literatura registra que os animais adultos apresentam-se mais resistentes às convulsões induzidas por pilocarpina em altas doses que os animais jovens, embora seja um modelo útil para o estudo do processo convulsivo, fornecendo informações referentes ao estado epiléptico de forma semelhante à da epilepsia do lobo temporal, uma vez que a desordem neurológica parece ter características diferentes em função da idade (George e Kulkarni, 1996).

\section{Objetivos}

Determinar os níveis dos neurotransmissores estriatais durante o estado epiléptico em ratas adultas induzido por pilocarpina em alta dose.

\section{Material e métodos}

Ratas wistar adultas (2 meses de idade; fêmeas; $250 \mathrm{~g}$ a $280 \mathrm{~g} ; \mathrm{n}=20$ ), provenientes do Biotério Central da Universidade Federal do Ceará, foram tratadas com uma única dose de pilocarpina $(400 \mathrm{mg} / \mathrm{kg}$; s.c.; $\mathrm{n}=7)$ e observadas por 1 hora após a administração. Os animais-controles foram tratados com solução salina $(\mathrm{NaCl}$, $0,9 \%$; s.c.; $\mathrm{n}=13$ ). Durante todos os experimentos, os animais foram mantidos em gaiolas com no máximo seis animais, em condições ambientais semelhantes, com ciclos de alternância claro/escuro de 12 horas, recebendo ração-padrão do tipo Purina ${ }^{\circledR}$ e água ad libitum. Os animais que apresentam convulsão, estado epiléptico e sobreviveram ao tratamento durante o período de 1 hora de observação foram decapitados e imediatamente o corpo estriado foi dissecado sobre gelo para preparação do homogenato.

Os níveis cerebrais de monoaminas foram estudados em homogenatos a $10 \%$ preparados em tampão fosfato de sódio $(150 \mathrm{mM} ; \mathrm{pH} 7,4)$. Para a determinação de neurotransmissores dopamina (DA), serotonina (5HT) e seus metabólitos ácido di-hidroxifenilacético (DOPAC), ácido 4-hidroxi-3-metoxi-fenilacético (HVA), ácido 5-hidroxiindolacético (5-HIAA), foi utilizado um equipamento de HPLC da Shimadzu, modelo LCD-6A, com detecção amperométrica. Para a separação das monoaminas, foi usada uma coluna de fase reversa (Shim-Pack CLC-ODS, $25 \mathrm{~cm}$ ). A fase móvel foi preparada com ácido cítrico monoidratado $150 \mathrm{mM}$, octil sulfato de sódio $67 \mathrm{mM}$, tetraidrofurano $2 \%$, acetonitrila 45 , utilizando água deionizada. $\mathrm{O} \mathrm{pH}$ da fase móvel foi ajustado para 3,0 com NaOH $10 \mathrm{mM}$. A quantificação dos picos obtidos foi feita com o auxílio de uma curva-padrão. Valores absolutos foram corrigidos quanto a recuperação das cânulas e das expressões de variação em relação aos valores basais e os resultados expressos em $\mathrm{ng} / \mathrm{g}$ de tecido.

Para estatística dos dados foi utilizada a análise de variância (ANOVA) para múltiplas comparações e o teste $\mathrm{t}$ de Student como teste post hoc. As diferenças foram consideradas estatisticamente significativas para $\mathrm{p}<0,05$. 


\section{Resultados}

A figura 1 mostra que a dose convulsiva de pilocarpina diminuiu de maneira significativa os níveis de dopamina (DA) em $40 \%$ e do ácido dihidroxifenilacético (DOPAC) em 24\%. Por usa vez, a concentração do ácido 4-hidroxi-3-metoxi-fenilacético (HVA) não sofreu nenhuma alteração durante o estado epiléptico.

Foi observada também uma redução na concentração da serotonina (5-HT) em 30\% e um aumento na concentração do seu metabólito, o ácido 5-hidroxiindolacético (5-HIAA) nas mesmas condições de estudo.

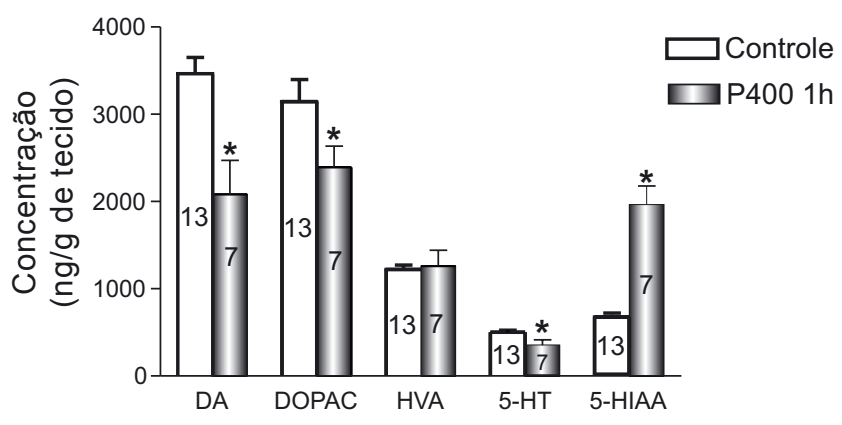

Monoaminas

Figura 1 Níveis dos neurotransmissores estriatais durante o estado epiléptico em ratas adultas convulsivas. Os animais com 2 meses de idade foram tratados com uma única dose de pilocarpina $(400 \mathrm{mg} / \mathrm{kg}$; s.c.; $\mathrm{P} 400 \mathrm{lh} ; \mathrm{n}=7$ ) e os controles com salina ( $\mathrm{NaCl} 0,9 \%$; s.c.; $\mathrm{n}=13)$, sendo observados e sacrificados 1 hora após a administração. Os valores foram expressos como média (ng/g de tecido) + EPM e o número de animais está inserido na barra. Abreviações: P400 1h (rata convulsiva); DA (dopamina), DOPAC (ácido diidroxifenilacético), HVA (ácido 4hidroxi-3-metoxi-fenilacético), 5-HT (serotonina) e o 5-HIAA (ácido 5 -hidroxiindolacético). ${ }^{*} \mathrm{p}<0.05$, controle versus pilocarpina (ANOVA e teste t-Student post hoc)

\section{Discussão}

A pilocarpina promove convulsões nos animais adultos que progridem na maior parte dos animais para o estado epiléptico. Os animais que apresentaram convulsões, estado epiléptico e sobreviveram até a primeira hora de observação, foram estudados quanto as possíveis mudanças na concentração dos neurotransmissores durante a fase aguda do processo convulsivo. Foram observados redução nos níveis de DA, 5-HT, DOPAC e aumento na concentração de 5-HIAA, e nenhuma alteração no HVA foi detectada durante o estudo.

Inúmeros estudos têm dado ênfase ao efeito epileptogênico da administração de alta dose de pilocarpina em ratos adultos, na intenção de verificar os efeitos de doses convulsivas nos animais desenvolvidos (Hirsch et al., 1992; Cavalheiro et al., 1987 e 1994). Esses estudos investigam também a origem e a propagação das convulsões no cérebro maduro, a fim de esclarecer a participação dos neurotransmissores durante o estado epiléptico.

Nossos dados revelaram que o tratamento agudo com pilocarpina não produz nenhuma morte durante a primeira hora da fase aguda da convulsão. O mesmo fato parece não ocorrer nos ratos jovens, pois modificações no percentual de morte, nessas mesmas condições, foram observadas (dados não mostrados), demonstrando que os animais adultos apresentam menor taxa de mortalidade. Várias pesquisas têm sugerido que na fase aguda das convulsões ocorre uma relação quase que imediata da acetilcolina com outros sistemas de neurotransmissão (adrenérgico, dopaminérgico, serotonérgico, glutamatérgico e GABAérgico), uma vez que efeitos rápidos nas concentrações de seus neurotransmissores têm sido determinados (El-Etri et al., 1993; Fritschy et al., 1999). Nos ratos adultos, em particular, os sistemas de neurotransmissão operantes podem promover ações semelhantes no sistema colinérgico durante o estado epiléptico e, com relação ao tempo, pode aumentar a participação de outros mecanismos para a propagação e/ou manutenção da ativação colinérgica, provavelmente já existentes no cérebro maduro.

Como relatado anteriormente, os níveis de monoaminas e metabólitos durante o estado epiléptico foram alterados nas ratas com 2 meses de idade, apresentando redução nos níveis de DA, DOPAC e 5-HT e aumento na concentração do 5-HIAA. O metabólito da dopamina HVA teve sua concentração inalterada, talvez em decorrência de um curto período de observação. Esses dados corroboram com os de El-Etri et al., (1993) somente no aumento da concentração do 5-HIAA, pois os pesquisadores observaram que os níveis de DA e 5HT não se alteraram, enquanto seus metabólitos sofreram um aumento significativo nas suas concentrações em ratos adultos convulsivos, após 1 hora de observação. Os dados de Cavalheiro et al. (1994) mostraram que houve um aumento no conteúdo de DA, no hipocampo durante o período agudo, silencioso e crônico das convulsões. Nossos dados para área estudada, o corpo estriado, não concordam com esses, pois observou-se redução na concentração de dopamina na primeira hora da fase aguda. A diminuição observada na concentração da 5-HT durante o estado epiléptico pode ser útil na manutenção das convulsões, uma vez que fisiologicamente a 5-HT inibe a estimulação colinérgica por meio da ativação de receptores do tipo 5-HT1A, com a finalidade de cessar ou inibir a convulsão.

Nossos achados apontaram para alterações nos neurotransmissores, exceto para o HVA, durante a fase 
aguda das convulsões, na primeira hora, indicando que, após a ativação colinérgica, sabidamente necessária para desencadear o processo convulsivo (Cavalheiro et al., 1987; Ramsay, 1993), os níveis dos neurotransmissores são modificados de diferentes formas e podem variar em função da fase da convulsão, possivelmente em decorrência da ativação e/ou inibição dos sistemas de neurotransmissão.

\section{Referências bibliográficas}

Al-TAJir, G.; Starr, B.S.; Starr, M.S. - Proconvulsant Effect of SKF 38393 Mediated by Nigral D1 Receptors. Eur J Pharmacol 162: 245-251, 1990a.

Aminoff, M.J.; Simon, R.P. - Status Epilepticus. Causes, Clinical Features and Consequences in 98 Patients. Am J Med 62: 657-66, 1980.

Barone, P.; Palma, V.; Debartomolemeis, A.; Tedeschi, E.; Muscettola, G.; Campanella, G. - Dopamine D1 and D2 Receptors Mediate Opposite Functions in Seizures Induced by Lithium-pilocarpine. Eur J Pharmacol 195: 157-62, 1991.

Cavalheiro, E.A.; Delfrio, F.S.; Turski, W.A.; Calderezzo F.L.S.; Bortolotto, Z.A.A.; Turski, L. - The Susceptiblity of Rats to Pilocarpine-induced Seizures is Age-dependent. Dev Brain Res 37: 43-58, 1987.

Cavalheiro, E.A.; Fernandes, M.J.; Turski, L.; Naffah-Mazzacoratti, M.G. - Spontaneous Recurrent Seizures in Rats: Amino Acid and Monoamine Determination in the Hippocampus. Epilepsia 35: 111, 1994.

Clifford, D.B.; Olney, J.W.; Maniotis, A.; Collins, R.C.; Zorumski, C.F. - The Functional Anatomy and Pathology of Lithium-pilocarpine and High-dose Pilocarpine Seizures. Neuroscience 23: 953-68, 1987.

Costa-lotufo, L.V.; Fonteles, M.M.F.; Lima, I.S.P.; Oliveira, A.A.; Nascimento, V.S.; Bruin, V.M.S.; Viana, G.S.B. - Attenuating Effects of Melatonin on Pilocarpine-induced Seizures in Rats. Comp Biochem Physiol C Pharmacol Toxicol Endocrinol 131: 521-9, 2002.

El-Etri, M.M.; EniIs, M.; JiAng, M.; Shipley, M.T. - Pilocarpine-induced Convulsions in Rats: Evidence for Muscarinic Receptor-mediated
Activation of Locus Coeruleus and Norepinephrine Release in Cholinolytic Seizure Development. Exp Neurol 121: 24-39, 1993.

Erakovic, V.; Zupan, G.; Varljen, J.; Laginja, J.; Simonic, A. - Lithium plus Pilocarpine Induced Status Epilepticus - Biochemical Changes. Neurosc Res 36: 157-66, 2000.

Fritschy, J.M.; Kiener, T.; Bouilleret, V.; Loup, F. - GABAergic Neurons and GABAa-receptors in Temporal Lobe Epilepsy. Neurochem Int 34: 435-45, 1999.

Fujikawa, D.G.; Daniels, A.H.; Kim, J.S. - The Competitive NMDA Receptor Antagonist CGP 40116 Protects Against Status EpilepticusInduced Neuronal Damage. Epilepsy Res 17: 207-19, 1994.

FuJIKAWA, D.G. - Neuroprotective Effect of Ketamine Administered After Status Epilepticus Onset. Epilepsia 36: 186-95, 1995.

George, B.; Kulkarni, S.K. - Protective Effects of GABAergic Drugs and Other Anticonvulsants in Llithium-pilocarpine-induced Status Epilepticus. Methods Find Exp Clin Pharmacol 18: 335-40, 1996.

Hirsch, E.; Baram, T.Z.; Snead III, O.C. - Ontogenic Study of Lithiumpilocarpine Induced Status Epilepticus in Rats. Brain Res 583:1206, 1992.

Khan, G.M.; Smolders, I.; Ebinger, G.; Мichotte, Y. - Anticonvulsant Effect and Neurotransmitter Modulation of Focal and Systemic 2chloroadenosine Against the Development of Pilocarpine-inducd Seizure. Neuropharmacology 39: 2418-32, 2000.

Marinho, M.M.F.; Sousa, F.C.F.; Bruin, V.M.S.; Aguiar, L.M.V.; Pinho, R.S.N.; VIANA, G.S.B. - Inhibitory Action of a Calcium Channel Blocker (nimodipine) on Seizures and Brain Damage Induced by Pilocarpine and Lithium-pilocarpine in Rats. Neuroscience Lett 235: 13-6, 1997.

Marinho, M.M.F.; Sousa, F.C.F.; Bruin, V.M.S.; Viana, G.S.B.- Effects of Lithium, Alone or Associated with Pilocarpine, on Muscarinic and Dopaminergic Receptors and on Phosphoinositide Metabolism in Rat Hippocampus and Striatum. Neurochem Int 33: 299-306, 1998.

Persinger, M.A.; Bureau, Y.R.J.; Kostakos, M.; Peredery; Falter, H. - Behaviours of Rats with Insidious Multifocal Brain Damage Induced by Seizures Following Single Peripheral Injections of Lithium and Pilocarpine. Physiol Behav 53: 849-66, 1993.

Turski, L.; IKonomidou, C.; Turski, W.A.; Bortolotto, Z.A.; CaValheiro, E.A. - Cholinergic Mechanisms and Epileptogenesis. The Seizures Induced by Pilocarpine: a Novel Experimental Model of Intractable Epilepsy. Synapse 3: 154-71, 1989.

Ramsay, R.E. - Treatment of status epilepticus. Epilepsia 34:71-81, 1993. 